\title{
Inhibition of IL-32 and TSLP production through the attenuation of caspase- 1 activation in an animal model of allergic rhinitis by Naju Jjok (Polygonum tinctorium)
}

\author{
HYUN-JA JEONG ${ }^{1}$, HYUN-A OH ${ }^{2}$, BYUNG-JOO LEE ${ }^{2}$ and HYUNG-MIN KIM ${ }^{2}$ \\ ${ }^{1}$ Biochip Research Center and Inflammatory Diseases Research Center, Hoseo University, Asan, Chungnam 336-795; \\ ${ }^{2}$ Department of Pharmacology, College of Korean Medicine, Kyung Hee University, Seoul 130-701, Republic of Korea
}

Received July 27, 2013; Accepted October 18, 2013

DOI: $10.3892 /$ ijmm.2013.1548

\begin{abstract}
In this study, we investigated the effects of Naju Jjok (Polygonum tinctorium Lour., NJJ) on interleukin (IL)-32 and thymic stromal lymphopoietin (TSLP) levels associated with allergic rhinitis (AR). Using female BALB/c mice, we created an animal model of ovalbumin (OVA)-induced AR. Prior to the callenge with OVA, the mice were administered, either nasally or orally with NJJ. In addition, we also used the eosinophilic cells line, Eol-1, stimulated with granulocyte-macrophage colony-stimulation factor (GM-CSF). The mRNA and protein levels of inflammatory cytokines and markers [interleukin (IL)-32, IL-4, macrophage-inflammatory protein-2 (MIP-2), intercellular adhesion molecule-1 (ICAM-1), and cyclooxygenase-2 (COX-2)] were measured by RT-PCR and western blot analysis, respectively and serum levels were measured by ELISA. The increased levels of IL-32 in the mice with AR and in the stimulated eosinophilic cell line, Eol-1, were significantly reduced by NJJ. TSLP levels were also decreased following the oral administration of NJJ. Mice orally administered NJJ showed markedly alleviated clinical symptoms, such as a reduced number of nasal rubs, decreased spleen weight, decreased serum immunoglobulin E (IgE) levels and decreased serum histamine levels. The oral administration of NJJ significantly decreased the IL-4 levels, while increasing the interferon- $\gamma$ levels in the spleen. The increased number of eosinophils and mast cells infiltrating the nasal mucosal tissue of the mice with AR were decreased following the oral administration of NJJ. NJJ effectively attenuated caspase-1 activity in the mice with AR and in the stimulated Eol-1 cells. The oral administration of NJJ significantly reduced the levels of inflammatory markers, such as MIP-2, ICAM-1 and COX-2. Furthermore, the intranasal administration of NJJ significantly
\end{abstract}

Correspondence to: Professor Hyung-Min Kim, Department of Pharmacology, College of Korean Medicine, Kyung Hee University, Seoul 130-701, Republic of Korea

E-mail: hmkim@khu.ac.kr

Key words: Naju Jjok, Polygonum tinctorium Lour., allergic rhinitis, interleukin-32, thymic stromal lymphopoietin reduced the early phase response to allergen exposure, such as nasal rubs, IgE production and histamine release, as well as the late phase responses, such as the expression of inflammatory markers. In conclusion, these data demonstrate that NJJ may play a regulatory role in nasal inflammation.

\section{Introduction}

Allergic rhinitis (AR), a very common chronic illness that affects patients of all ages, initiates the allergic reaction induced by the release of preformed mediators and the generation of inflammatory mediators from mast cells and eosinophils (1). The levels of interleukin (IL)-1, IL-5, IL-6, macrophage-inflammatory protein-2 (MIP-2), and granulocyte-macrophage colony-stimulation factor (GM-CSF) are increased during the early and late phase of $\operatorname{AR}(2,3)$. The cytokine messenger RNA (mRNA) expression of IL-3, IL-4, IL-5 and GM-CSF has been shown to be increased in the nasal mucosa of patients with AR following allergen provocation and is associated with tissue eosinophilia (4). Activated endothelial cells express intercellular adhesion molecule-1 (ICAM-1) and vascular cell adhesion molecule-1 (VCAM-1) on their cell surfaces (5). GM-CSF is an important activating factor for eosinophils and neutrophils, and is known as a pleiotropic and pro-inflammatory cytokine (6). GM-CSF induced the expression of IL-32 in eosinophils (7).

IL-32 is associated with AR, cancer, infection and chronic inflammation (7,8-10). IL-32 is a cytokine produced mainly by immune cells, including $\mathrm{T}$ lymphocytes, natural killer cells, epithelial cells, mast cells, keratinocytes, eosinophils and blood monocytes $(11,12)$. IL-32 has previously been shown to contribute to pro-inflammatory cytokine synthesis (7). Several pathways have been shown to be involved in inflammatory processes, including the phosphatidylinositide 3-kinase (PI3K)/Akt pathway, nuclear factor (NF)- $\mathrm{B} / \mathrm{AP}-1$ pathway, p38 mitogen-activated protein kinase (MAPK) and caspase-1 pathways $(7,12,13)$. Viral-induced IL-32 expression seems to be mediated by cyclooxygenase-2 (COX-2) pathways (14).

Thymic stromal lymphopoietin (TSLP) is a central factor in allergic inflammation and allergy-related diseases, including atopic dermatitis (AD), asthma and AR (15-17). TSLP is expressed and produced by caspase -1 and $N F-\kappa B$ in mast cells $(18,19)$. Recently, we reported that IL-32 induced TSLP 
production through the activation of NF- $\kappa \mathrm{B}$ and caspase- 1 in monocytes (20).

Traditional Korean medicine (TKM) has been used for thousands of years. Naju Jjok (Polygonum tinctorium Lour., NJJ) is a species of flowering plant in the buckwheat family (commonly known as Chinese indigo) and has been used for its detoxifying, antibacterial, anticancer, antioxidant, anti-inflammatory and anti-allergic properties (21-24) and traditionally, as a textile dye. Tryptanthrin, kaempferol and indirubin are the main components of NJJ. Tryptanthrin and kaempferol have antibacterial properties (23). Kaempferol has been shown to inhibit the production of IL-8 and tumor necrosis factor (TNF)- $\alpha$ and the infiltration of eosinophils in allergic reactions $(41,42)$. Indirubin has been shown to inhibit inflammatory reactions and allergic contact dermatitis $(43,44)$ However, the effects of NJJ on AR have not yet been fully elucidated. In this study, we evaluated the anti-allergic and anti-inflammatory effects of NJJ on mice with ovalbumin (OVA)-induced AR, as well as on the GM-CSFstimulated human eosinophilic cell line, Eol-1.

\section{Materials and methods}

Materials. OVA, $o$-phthaldialdehyde (OPA), avidin peroxidase (AP), 2,2'-azino-bis(3-ethylbenzthiazoline-6-sulfonic acid) substrate tablets (ABTS), 3-(4,5-dimethylthiazol-2-yl)2,5-diphenyltetrazolium bromide (MTT) and bicinchoninic acid (BCA) were purchased from Sigma (St. Louis, MO, USA). Fetal bovine serum (FBS), Roswell Park Memorial Institute (RPMI)-1640, and streptomycin were purchased from Gibco-BRL (Grand Island, NY, USA). Anti-mouse immunoglobulin E(IgE)/TSLP/IL-4/interferon(IFN)- $\gamma /$ MIP-2/ICAM-1 antibody $(\mathrm{Ab})$, biotinylated anti-mouse IgE/TSLP/IL-4/IFN- $\gamma /$ MIP-2/ICAM-1 Ab, recombinant mouse IgE/TSLP/IL-4/ IFN- $\gamma /$ MIP-2/ICAM-1, anti-human IL-8 Ab, biotinylated antihuman IL-8 Ab and recombinant human IL-8/GM-CSF were purchased from Pharmingen (San Diego, CA, USA). The IL-32 Abs was obtained from BioLegend (San Diego, CA, USA) and Acris (Herford, Germany). Abs against COX-2, caspase-1 and actin were obtained from Santa Cruz Biotechnology, Inc. (Santa Cruz, CA, USA). The caspase-1 assay kit was supplied by R\&D Systems Inc. (Minneapolis, MN, USA).

Preparation of NJJ. NJJ was obtained from Naju (Korea). The whole plant material of NJJ was extracted with distilled water (DW) at $80^{\circ} \mathrm{C}$ for $3 \mathrm{~h}$. The filtered crude extracts were lyophilized and reduced to powder. The yield of dried extract from the starting materials was approximately $18 \%(\mathrm{w} / \mathrm{w})$. The NJJ was dissolved in DW and filtered with a $0.22-\mu \mathrm{m}$ syringe filter.

OVA-induced animal model of AR. We maintained 6-weekold female BALB/c mice (Charles River Laboratories, Inc., Wilmington, MA, USA) under pathogen-free conditions. Mouse care and experimental procedures were performed under the approval of the Animal Care and Use Committee of Kyung Hee University [KHUASP(SE)-11-037]. We sensitized the mice on days 1,5 and 14 by an intraperitoneal (i.p.) injection of $100 \mu \mathrm{g}$ OVA emulsified in $20 \mathrm{mg}$ aluminum hydroxide (Sigma) and then challenged the mice with $1.5 \mathrm{mg}$ OVA. NJJ was orally $(1,10$, or $100 \mathrm{mg} / \mathrm{kg}$ ) or intranasally (i.n., $2 \mu \mathrm{l}$ of $40 \mu \mathrm{g} /$ nostril) administered prior to i.n. OVA challenge for
10 days. Nasal symptoms were evaluated by counting the number of nasal rubs that occurred in the $10 \mathrm{~min}$ following OVA i.n. provocation. The mice were divided into 3 groups: i) the normal group, in which the mice were not sensitized/ challenged with OVA; ii) the control group, in which the mice were sensitized/challenged with OVA; and the iii) the NJJ group, in which the mice were sensitized/challenged with OVA and administered NJJ. There were 5 mice in each group.

Enzyme-linked immunosorbent assay (ELISA). The Eol-1 cells $\left(3 \times 10^{5}\right)$ were treated with $\mathrm{NJJ}(1,10$, or $100 \mu \mathrm{g} / \mathrm{ml})$ for $1 \mathrm{~h}$ prior to stimulation with GM-CSF and incubated for $24 \mathrm{~h}$. Cytokines in serum, nasal mucosal tissue and spleen tissue, as well as in the cell supernatants were measured by ELISA. ELISA was performed by coating 96-well plates with $1 \mathrm{mg} /$ well of capture $\mathrm{Ab}$. Before the subsequent steps in the assay, the coated plates were washed twice with $1 \mathrm{X}$ PBS containing $0.05 \%$ Tween-20 (PBST). All reagents and coated wells used in this assay were incubated for $2 \mathrm{~h}$ at room temperature. The standard curve was generated from known concentrations of cytokines, as provided by the manufacturer. Following exposure to the medium, the assay plates were exposed sequentially to each of the biotin-conjugated secondary antibodies, as well as AP and ABTS substrate solution containing $30 \% \mathrm{H}_{2} \mathrm{O}_{2}$. The plates were read at an absorbance of $405 \mathrm{~nm}$. IL-32 was analyzed according to the manufacturer's specifications. Appropriate specificity controls were included, and all samples were run in duplicate. Cytokine levels in the spleen and nasal mucosa were divided according to the total protein levels. Protein levels were determined using a BCA kit.

Histamine assay. Histamine serum levels were measured by the OPA spectrofluorometric procedure. The fluorescent intensity was measured at $440 \mathrm{~nm}$ (excitation at $360 \mathrm{~nm}$ ) using a spectrofluorometer.

Histological examination. Mice were euthanized by carbon dioxide inhalation. Nasal mucosa tissues from the euthanized mice were removed. Tissue samples were immediately fixed with $10 \%$ formaldehyde and embedded in paraffin. The section of the nasal mucosa samples were $4-\mu$ m-thick. Each section was stained with hematoxylin and eosin (H\&E, for eosinophils) prior to dewaxing and dehydration. The number of eosinophils on both sides of the septal mucosa was counted. The sections were coded and randomly analyzed by 2 blinded observers.

Culture of Eol-1 cells. Human Eol-1 cells were a kind gift from Dr H. Bae (Kyung Hee University, Seoul, Korea). The Eol-1 cells were grown in RPMI-1640 supplemented with $100 \mathrm{U} / \mathrm{ml}$ penicillin, $100 \mathrm{mg} / \mathrm{ml}$ streptomycin and $10 \%$ heat-inactivated FBS at $37^{\circ} \mathrm{C} 5 \% \mathrm{CO}_{2}$ and $95 \%$ humidity. The Eol-1 cells were treated with $\mathrm{NJJ}(1,10$, or $100 \mu \mathrm{g} / \mathrm{ml})$ for $1 \mathrm{~h}$ prior to stimulation with GM-CSF.

Reverse transcription-polymerase chain reaction (RT-PCR). Eol-1 cells $\left(3 \times 10^{6}\right)$ were treated with $\mathrm{NJJ}(1,10$, or $100 \mathrm{mg} / \mathrm{ml})$ for $1 \mathrm{~h}$ prior to stimulation with GM-CSF and incubated for $4 \mathrm{~h}$. Total RNA was isolated from the cells and nasal mucosal tissue according to the manufacturer's specifications using an easy-BLUE ${ }^{\mathrm{TM}}$ RNA extraction kit (iNtRON Biotech, Sungnam, 
A
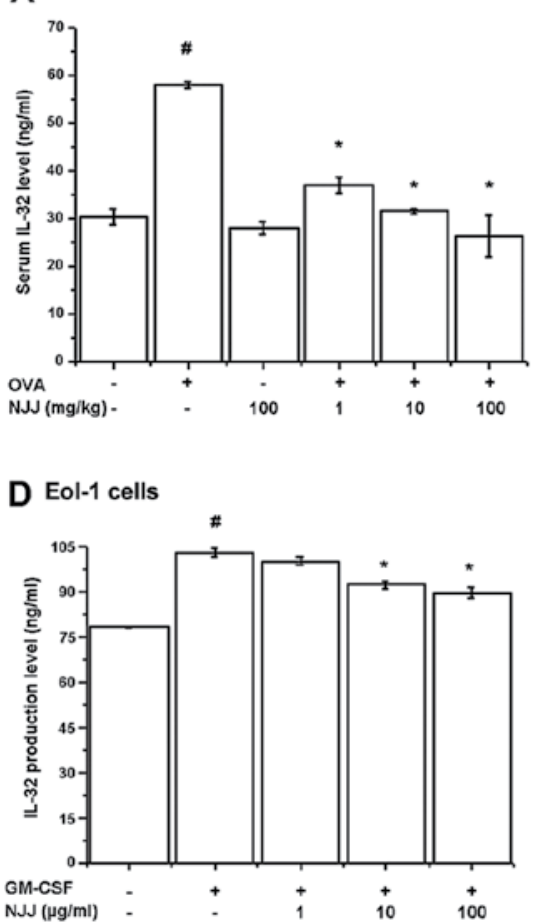

B

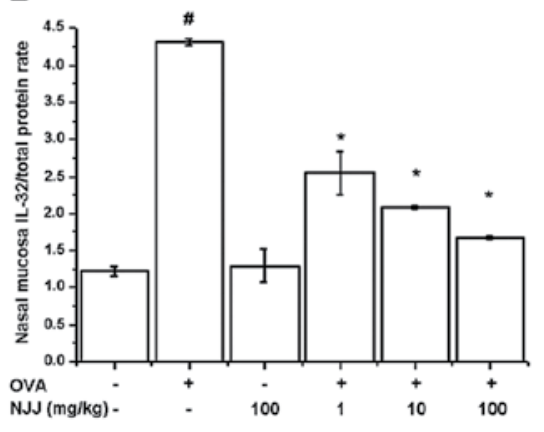

E Eol-1 cells

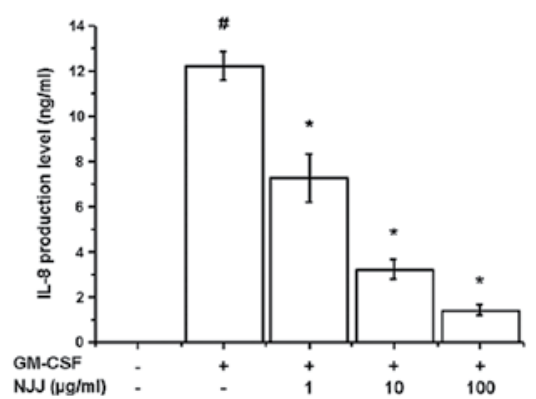

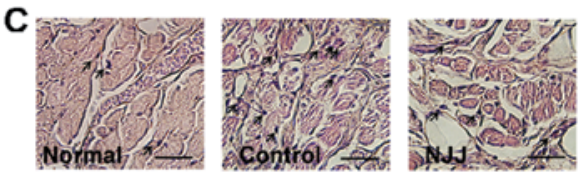

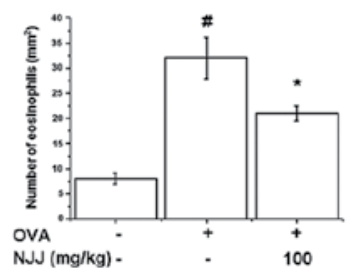

F Eol-1 cells

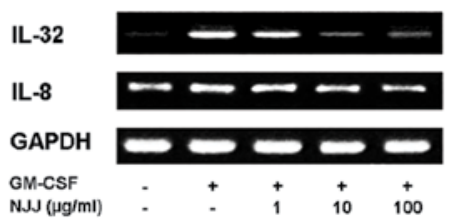

Figure 1. Effects of Naju Jjok (NJJ) on interleukin (IL)-32 levels. NJJ was administered orally for 10 days prior to the i.n. ovalbumin (OVA) challenge. (A and B) IL-32 levels were measured by ELISA in the serum and nasal mucosal tissue. (C) Nasal mucosal tissue was stained with hematoxylin and eosin (H\&E) for eosinophils (black arrow, upper panel). Five randomly selected tissue sections per mouse were counted (lower panel). The absolute number of cells was counted as the mean \pm SEM. ${ }^{\#} \mathrm{P}<0.05$, significantly different from the OVA-unsensitized mice. ${ }^{*} \mathrm{P}<0.05$, significantly different from the OVA-sensitized mice. Normal, OVA unsensitization; control, OVA sensitization; NJJ, OVA sensitization and NJJ (100 mg/kg) administration. (Original magnification, x400; scale bar, $50 \mu \mathrm{m})$. N=5. (D and E) Eol-1 cells were treated with NJJ $(1,10$, or $100 \mu \mathrm{g} / \mathrm{ml})$ and then stimulated with granulocyte-macrophage colony-stimulation factor (GM-CSF). IL-32 and IL-8 levels were measured by ELISA. (F) mRNA levels were measured by RT-PCR. ${ }^{\#} \mathrm{P}<0.05$, significantly different from the unstimulated cells. ${ }^{*} \mathrm{P}<0.05$, significantly different from the GM-CSF-stimulated cells.

Korea). The concentration of total eluted RNA was determined by spectrophotometry. Total RNA $(2.5 \mathrm{mg})$ was heated at $65^{\circ} \mathrm{C}$ for $10 \mathrm{~min}$ and then chilled on ice. Each sample was reversetranscribed to cDNA for $90 \mathrm{~min}$ at $37^{\circ} \mathrm{C}$ using the cDNA synthesis kit (Amersham Pharmacia Biotech, Piscataway, NJ, USA). PCR was performed using the following primers: mouse TSLP (5'-TAT GAG TGG GAC CAA AAG TAC CG-3' and 5'-GGG ATT GAA GGT TAG GCT CTG G-3'); mouse GAPDH (5'-TTC ACC ACC ATG GAG AAG GC-3' and 5'-GGC ATG GAC TGT GGT CAT GA-3'); human IL-8 (5'-CGA TGT CAG TGC ATA AAG ACA-3' and 5'-TGA ATT CTC AGC CCT CTT CAA AAA-3'); human IL-32 (5'-TGA CAT GAA GAA GCT GAA GGC-3' and 5'-CAT GAC CTT GTC ACA AAA GCT C-3'); and human GAPDH (5'-TCG ACA GTC AGC CGC ATC TTC TTT-3' and 5'-ACC AAA TCC GTT GAC TCC GAC CTT-3') was used to verify whether equal amounts of RNA were used for reverse transcription and PCR amplification under the different experimental conditions. The annealing temperature was $60^{\circ} \mathrm{C}$ for TSLP, IL- 8 , IL-32 and GAPDH. Products were electrophoresed on a $1.5 \%$ agarose gel and visualized by staining with ethidium bromide.

Confocal laser scanning microscopy. The tissue samples were fixed with $4 \%$ formaldehyde and embedded in paraffin. After dewaxing and dehydration, the sections were blocked with bovine serum albumin followed by $60 \mathrm{~min}$ of incubation with an anti-mouse c-Kit (Santa Cruz Biotechnology, Inc.) at a concentration of $1 \mu \mathrm{g} / \mathrm{ml}$. The secondary antibody, fluorescein isothiocyanate (FITC)-conjugated anti-mouse $\mathrm{IgG}$ (Invitrogen, Carlsbad, CA, USA), was added to the incubation medium for $30 \mathrm{~min}$. The mounting medium containing 4',6-diamidino-2-phenylinodole (DAPI; Vector Laboratories, Burlingame, CA, USA) was used to counterstain the DNA. All specimens were examined under a confocal laser-scanning microscope. The numbers of mast cells on both sides of the septal mucosa were counted. The sections were coded and randomly analyzed by 2 blinded observers.

Western blot analysis. Western blot analysis was used to analyze the nasal mucosal tissue extracts which were prepared by the detergent lysis procedure. Samples were heated at $95^{\circ} \mathrm{C}$ for $5 \mathrm{~min}$, and briefly cooled on ice. Following centrifugation at $15,000 \mathrm{x} \mathrm{g}$ for $5 \mathrm{~min}, 50 \mathrm{mg}$ aliquots were resolved by $12 \%$ SDS-PAGE. Resolved proteins were electrotransferred overnight onto nitrocellulose membranes in $25 \mathrm{mM}$ Tris, $\mathrm{pH}$ 8.5, $200 \mathrm{mM}$ glycerin, $20 \%$ methanol at $25 \mathrm{~V}$. The blots were blocked for at least $2 \mathrm{~h}$ with PBST containing 5\% non-fat dry milk and then incubated with primary antibodies for $1 \mathrm{~h}$ at room temperature. Blots were developed by peroxidase-conjugated secondary antibodies, and proteins were visualized by enhanced chemiluminescence procedures (Amersham Biosciences, Piscataway, NJ, USA) according to the manufacturer's instructions. 
A

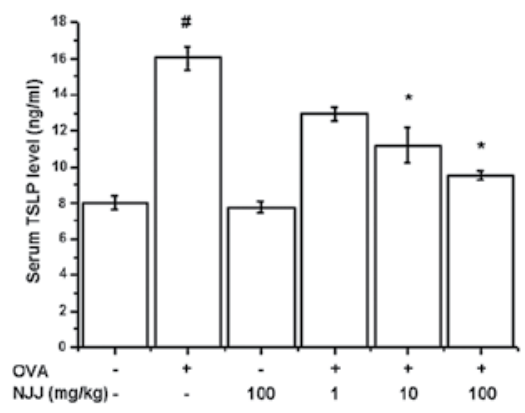

B

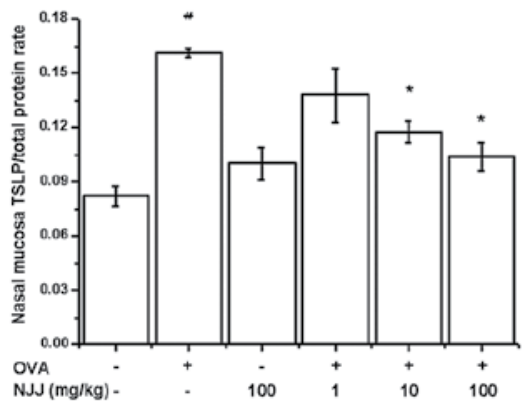

C

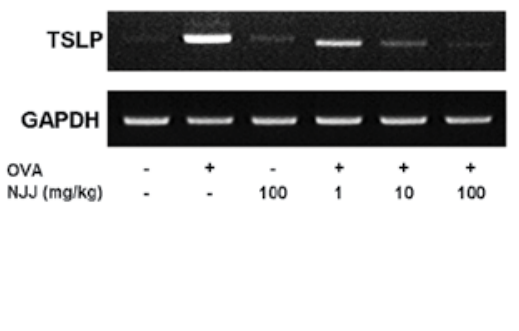

E

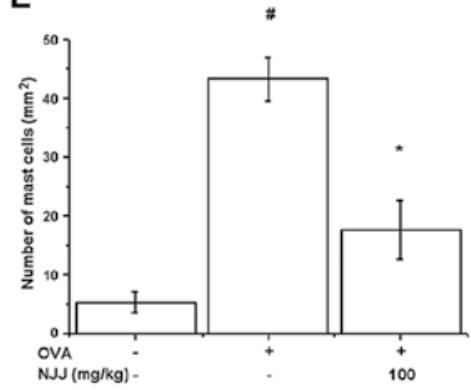

Figure 2. Effects of Naju Jjok (NJJ) on thymic stromal lymphopoietin (TSLP) levels. NJJ was administered orally for 10 days prior to i.n. ovalbumin (OVA) challenge. (A and B) TSLP levels were measured by ELISA in the serum and nasal mucosal tissue. (C) mRNA was measured by RT-PCR. All parameters measured in the tissue homogenates were presented as a ratio to the total protein levels in the tissue. (D) Mast cells were stained by a primary anti-c-Kit for $1 \mathrm{~h}$ and then incubated with secondary FITC-conjugated IgG for $1 \mathrm{~h}$ (white arrows). (E) Mast cells were counted by 3 individuals. Five randomly selected tissue sections per mouse were counted. The absolute number of cells was counted as the mean \pm SEM. ${ }^{\# P}<0.05 \mathrm{~m}$, significantly different from the OVA-unsensitized mice. "P<0.05, significantly different from the OVA-sensitized mice. Normal, OVA unsensitization; control, OVA sensitization; NJJ, OVA sensitization and NJJ $(100 \mathrm{mg} / \mathrm{kg}$ ) administration. (Original magnification, $\mathrm{x} 600$; scale bar, $50 \mu \mathrm{m}) . \mathrm{N}=5$.

Caspase-1 assay. The Eol-1 cells $\left(3 \times 10^{6}\right)$ were treated with NJJ $(1,10$ or $100 \mu \mathrm{g} / \mathrm{ml})$ for $1 \mathrm{~h}$ prior to stimulation with GM-CSF and incubated for $2 \mathrm{~h}$. Caspase- 1 assay was used to analyze the nasal mucosal tissue and cell extracts. Caspase-1 activity was measured according to the manufacturer's specifications using the caspase assay kit (R\&D Systems Inc.). Equal amounts of total protein were quantified by the BCA protein quantification kit (Sigma) in each lysate. The catalytic activity of caspase-1 from the cell lysates was measured by the proteolytic cleavage of WEHD-pNA for $4 \mathrm{~h}$ at $37^{\circ} \mathrm{C}$. The plates were read at $405 \mathrm{~nm}$.

MTT assay. The Eol-1 cells $\left(2 \times 10^{5}\right.$ cells $\left./ \mathrm{ml}\right)$ were cultured in microplate wells for $24 \mathrm{~h}$ following treatment with $\mathrm{NJJ}$ (1, 10 , or $100 \mu \mathrm{g} / \mathrm{ml}$ ) and incubated with $20 \mu \mathrm{l}$ of MTT solution $(5 \mathrm{mg} / \mathrm{ml})$ for an additional $4 \mathrm{~h}$ at $37^{\circ} \mathrm{C}$ in an atmosphere of $5 \%$ $\mathrm{CO}_{2}$ and $95 \%$ air. Consecutively, $250 \mu \mathrm{l}$ of DMSO were added to extract the MTT formazan and the absorbance of each well at $540 \mathrm{~nm}$ was read using an automatic microplate reader.

Statistical analysis. The experiments shown are a summary of the data from at least 3 experiments and statistical analyses were performed using SPSS statistical software (SPSS 11.5; SPSS Inc., Chicago, USA). The effects of treatment were analyzed by one-way ANOVA and Tukey's multiple range tests, and a value of $\mathrm{P}<0.05$ was used to indicate a statistically significant difference.

\section{Results}

Effects of NJJ on IL-32 levels. Recently, we reported that IL-32 is an important factor in patients and animals with AR and is expressed in eosinophils (7). Thus, in this study, we examined the effects of NJJ on IL-32 levels in animal models of AR and GM-CSF-stimulated Eol-1 cells. IL-32 levels in serum and nasal mucosal tissues of mice with AR were significantly increased compared with those of normal mice (Fig. 1A and B). However, the increased IL-32 levels significantly decreased following the administration of NJJ (Fig. 1A and B). Eosinophil infiltration increased following OVA challenge and significantly decreased following the administration of NJJ (Fig. 1C). To assess the regulatory effects of NJJ on IL-32 expression in the in vitro model, we measured IL-32 production and mRNA expression in GM-CSF-stimulated Eol-1 cells. The protein and mRNA levels of IL-32 were significantly inhibited by treatment with NJJ (Fig. 1D and F). IL-8 production was analyzed to examine the activity of GM-CSF. IL- 8 production and mRNA expression were decreased following treatment with NJJ (Fig. 1E and F). NJJ had no effect on cell viability (data not shown).

Effect of NJJ on TSLP levels. TSLP is expressed in the nasal mucosa of mice with AR (25) and is produced in activated mast 

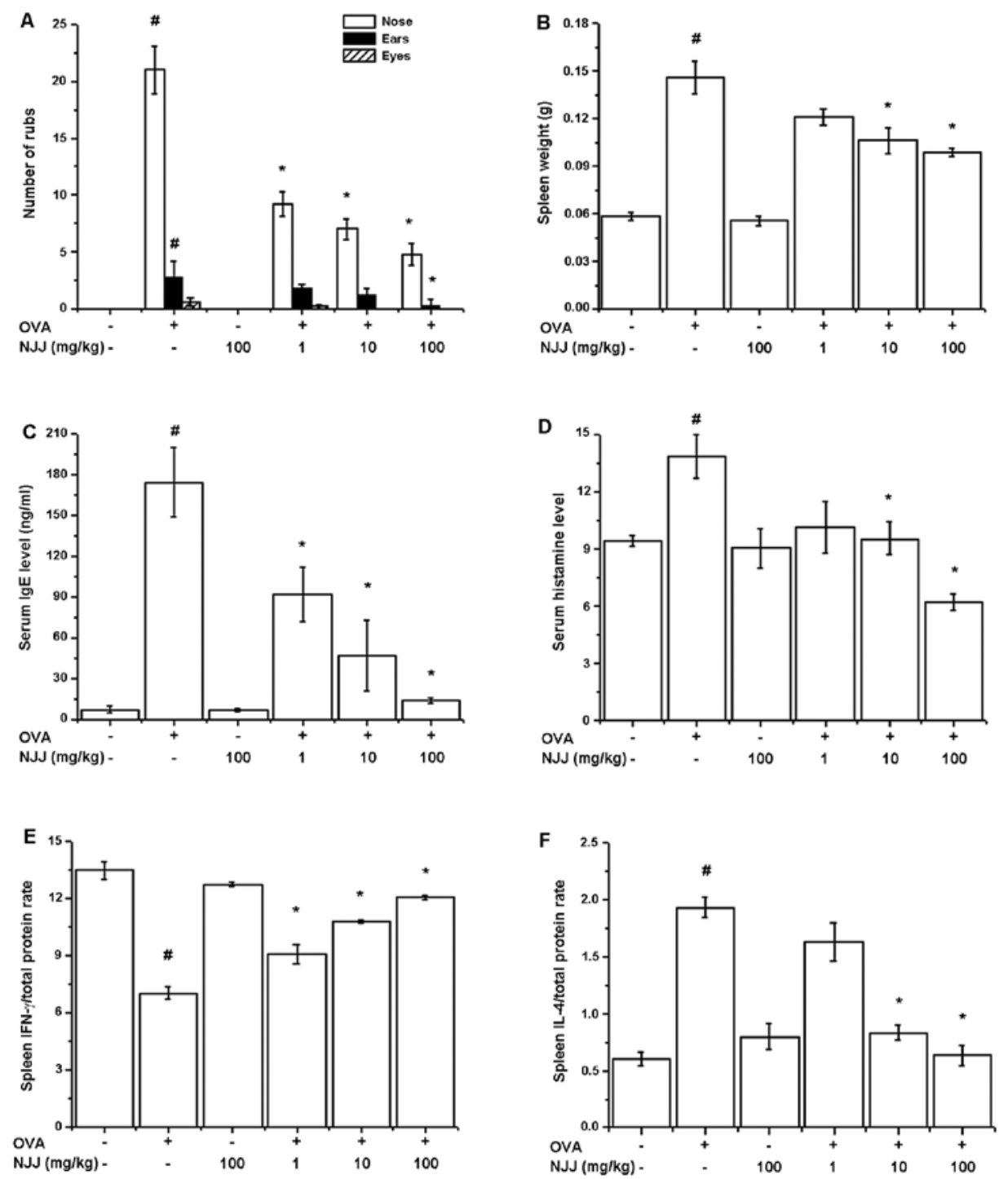

Figure 3. Effects of Naju Jjok (NJJ) in the clinical symptoms of mice with allergic rhinitis (AR). NJJ was administrated orally for 10 days prior to the i.n. ovalbumin (OVA) challenge. (A) Number of the nose, ear and eye rubs that occurred in the 10 min after OVA intranasal provocation. (B) Spleen weight. (C) Serum was isolated from blood and then assayed for IgE. (D) Serum histamine levels were measured by histamine assay. (E) IFN- $\gamma$ and (F) IL-4 in the spleen were measured by the ELISA. All parameters measured in the tissue homogenates were presented as a ratio to the total protein levels in the tissue. " $\mathrm{P}<0.05$, significantly different from the OVA-unsensitized mice. ${ }^{*} \mathrm{P}<0.05$, significantly different from the OVA-sensitized mice. $\mathrm{N}=5$.

cells (26). In our study, mice administered NJJ showed significantly decreased levels of TSLP in serum and nasal mucosal tissue (Fig. 2A and B). The oral administration of NJJ reduced TSLP mRNA expression (Fig. 2C). TSLP is expressed in mast cells $(18,19)$. Mast cells play a central role in AR (1). Thus, we determines whether the oral administration of NJJ suppresses mast cell infiltration in mice with AR. The respective numbers of mast cells in the nasal mucosa of the mice with AR were significantly higher than those in the OVA-unsensitized mice; however, the oral administration of NJJ significantly reduced the infiltration of mast cells (Fig. 2D and E).

Effect of oral administration of NJJ on clinical symptoms in mice with $A R$. To investigate the regulatory effects of NJJ in our animal model of AR, NJJ was administrated orally prior to OVA i.n. challenge for 10 days. The oral administration of NJJ significantly reduced the clinical symptoms (nasal rubs, spleen weight and levels of histamine, IFN- $\gamma$, IL-4 in spleen) in mice with AR (Fig. 3A). Spleen weight increased following challenge with OVA; this was reduced following the oral administration of NJJ (Fig. 3B). The IgE and histamine levels which were increased by OVA were also reduced by the oral administration of NJJ (Fig. 3C and D). To investigate the Th1/ $\mathrm{Th} 2$ immune reactions in mice administered NJJ, we measured the IFN- $\gamma$ and IL-4 levels in the spleen extracts. As shown in Fig. 3E and F, NJJ significantly increased IFN- $\gamma$ levels, while decreasing IL-4 levels compared with the control group.

Effects of NJJ on activation of caspase-1. To investigate the regulatory mechanisms of NJJ on IL-32 and TSLP production, caspase-1 assay and western blot analysis for caspase-1 were performed on the nasal mucosal tissues and GM-CSF-stimulated Eol-1 cell extracts. OVA-induced and GM-CSF-induced caspase-1 activities were decreased by NJJ (Fig. 4A and C). The active form of caspase-1 in the nasal mucosal tissue was reduced following the oral administration of NJJ (Fig. 4B). 


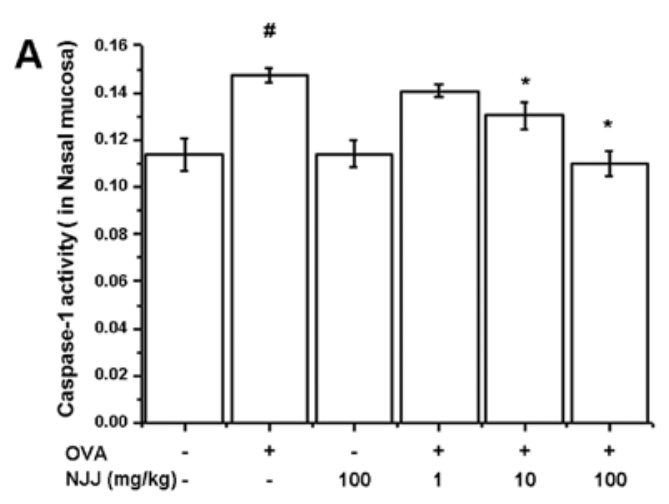

B
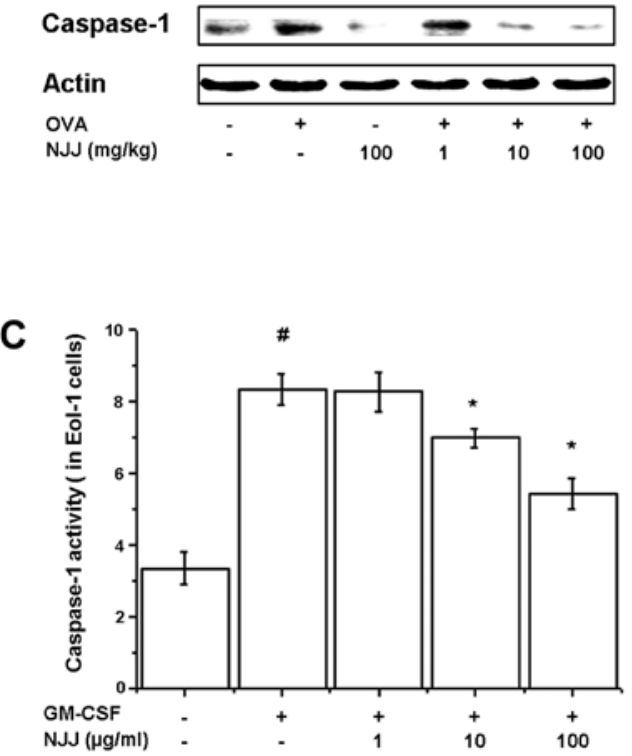

Figure 4. Effects of Naju Jjok (NJJ) on caspase-1 activation. (A) The enzymatic activity of caspase- 1 was examined by a caspase- 1 colorimetric assay in nasal mucosal tissue of mice with allergic rhinitis (AR). ${ }^{\text {" }} \mathrm{P}<0.05$, significantly different from the ovalbumin (OVA)-unsensitized mice. $\mathrm{N}=5$. ${ }^{*} \mathrm{P}<0.05$, significantly different from the OVA-sensitized mice. (B) Caspase-1 protein expression was evaluated by western blot analysis in nasal mucosal tissue of mice with AR. (C) Eol-1 cells were treated with NJJ (1, 10, or $100 \mu \mathrm{g} / \mathrm{ml})$ and then stimulated with granulocyte-macrophage colony-stimulation factor (GM-CSF) for $2 \mathrm{~h}$. The enzymatic activity of caspase-1 was examined by a caspase-1 colorimetric assay. ${ }^{\#} \mathrm{P}<0.05$, significantly different from the unstimulated cells. ${ }^{*} \mathrm{P}<0.05$, significantly different from the GM-CSF-stimulated cells

Effects of NJJ on inflammatory markers in mice with AR. To investigate the effects of NJJ on inflammation-related protein levels, we analyzed the MIP-2, ICAM-1 and COX-2 levels in the nasal mucosal tissue of mice with AR. The increased levels of MIP-2, ICAM-1 and COX-2 in the mice with AR were significantly reduced following the oral administration of NJJ (Fig. 5).

Effects of i.n. administration of NJJ on clinical symptoms in mice with $A R$. To investigate the effects of the i.n. administration of NJJ (IN-NJJ) on clinical symptoms in mice with AR, IN-NJJ was directly administered into the nasal cavity prior to OVA i.n. challenge for 10 days. The clinical symptoms (spleen weight, serum IgE and histamine levels, IFN- $\gamma$ levels, ICAM-1 levels) were decreased following IN-NJJ administration (Fig. 6A). The spleen weight was significantly decreased
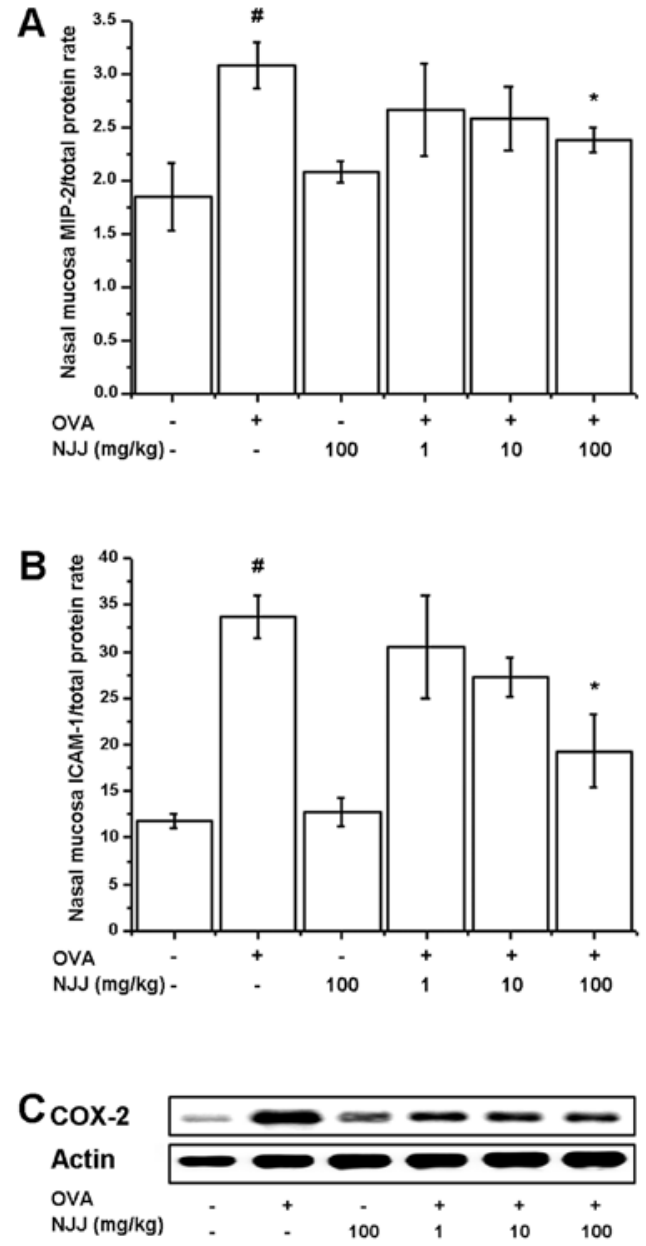

Figure 5. Effects of Naju Jjok (NJJ) on inflammatory markers. (A) Macrophageinflammatory protein-2 (MIP-2) and (B) intercellular adhesion molecule-1 (ICAM-1) levels were measured by ELISA in the nasal mucosal tissue. (C) Cyclooxygenase-2 (COX-2) protein expression was evaluated by western blot analysis. All parameters measured in the tissue homogenates were presented as a ratio to the total protein levels in the tissue. ${ }^{~} \mathrm{P}<0.05$, significantly different from the ovalbumin (OVA)-unsensitized mice. ${ }^{*} \mathrm{P}<0.05$, significantly different from the OVA-sensitized mice. $\mathrm{N}=5$.

following IN-NJJ administration (Fig. 6B). Serum IgE and histamine levels which were increased by OVA were reduced in the IN-NJJ-administered mice (Fig. 6C and D). As shown in Fig. 6E and F, IN-NJJ administration significantly increased the IFN- $\gamma$ levels, while decreasing the IL-4 levels compared with the control group. We measured the TSLP levels in the mice with AR. As shown in Fig. 6G, the IN-NJJ-administered mice showed significantly decreased levels of TSLP compared with the untreated mice with AR. TSLP mRNA levels were also significantly decreased following IN-NJJ administration (Fig. 6H). The levels of MIP-2, ICAM-1 and COX-2 in the nasal mucosal tissue were significantly reduced by IN-NJJ administration (Fig. 6I-K). In addition, caspapae-1 activity, which was increased by OVA was decreased following IN-NJJ administration (Fig. 6L).

\section{Discussion}

$\mathrm{AR}$ is an inflammatory disease of the nasal mucosa in which a number of cells and mediators play a part. These mediators may 

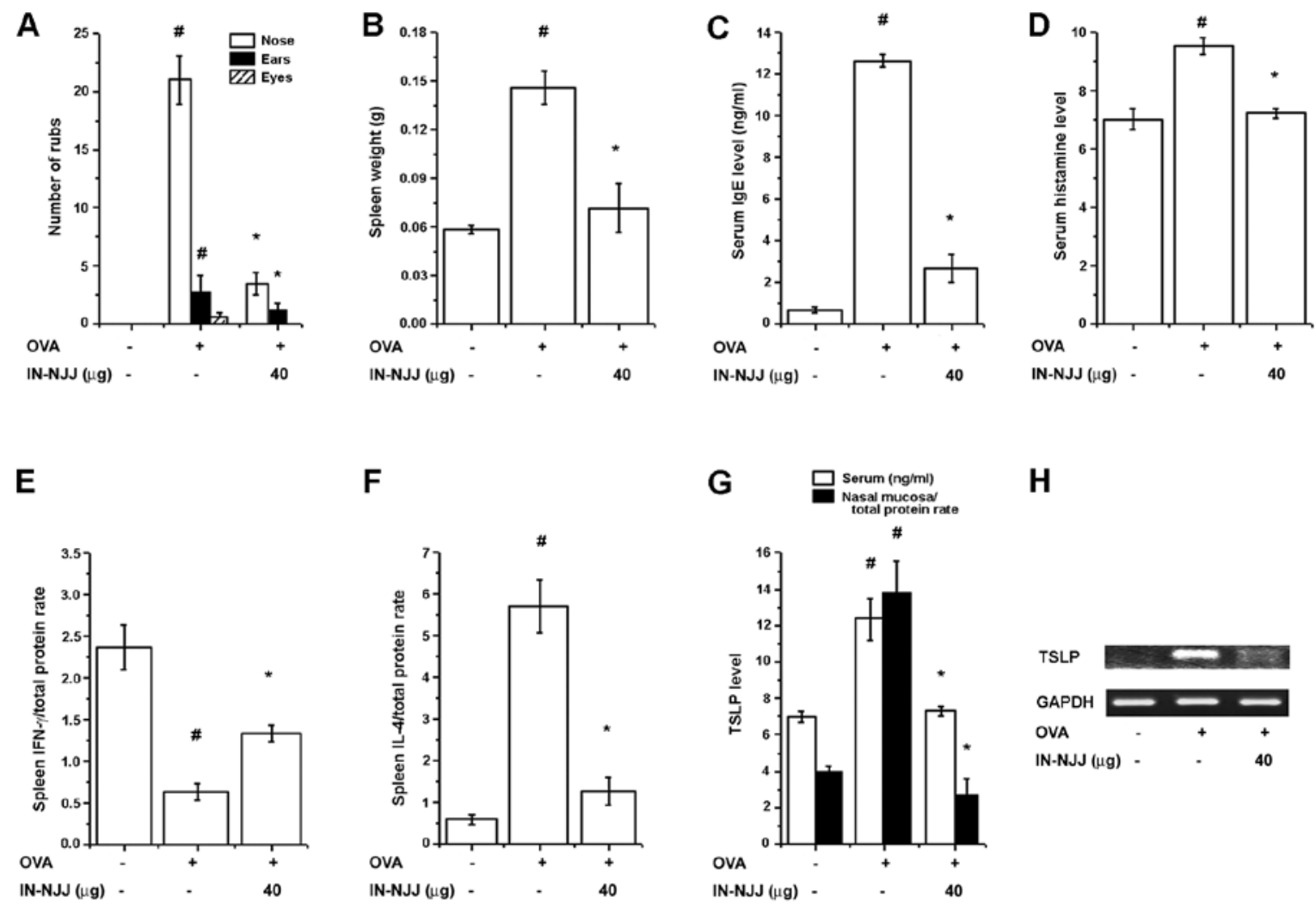

H
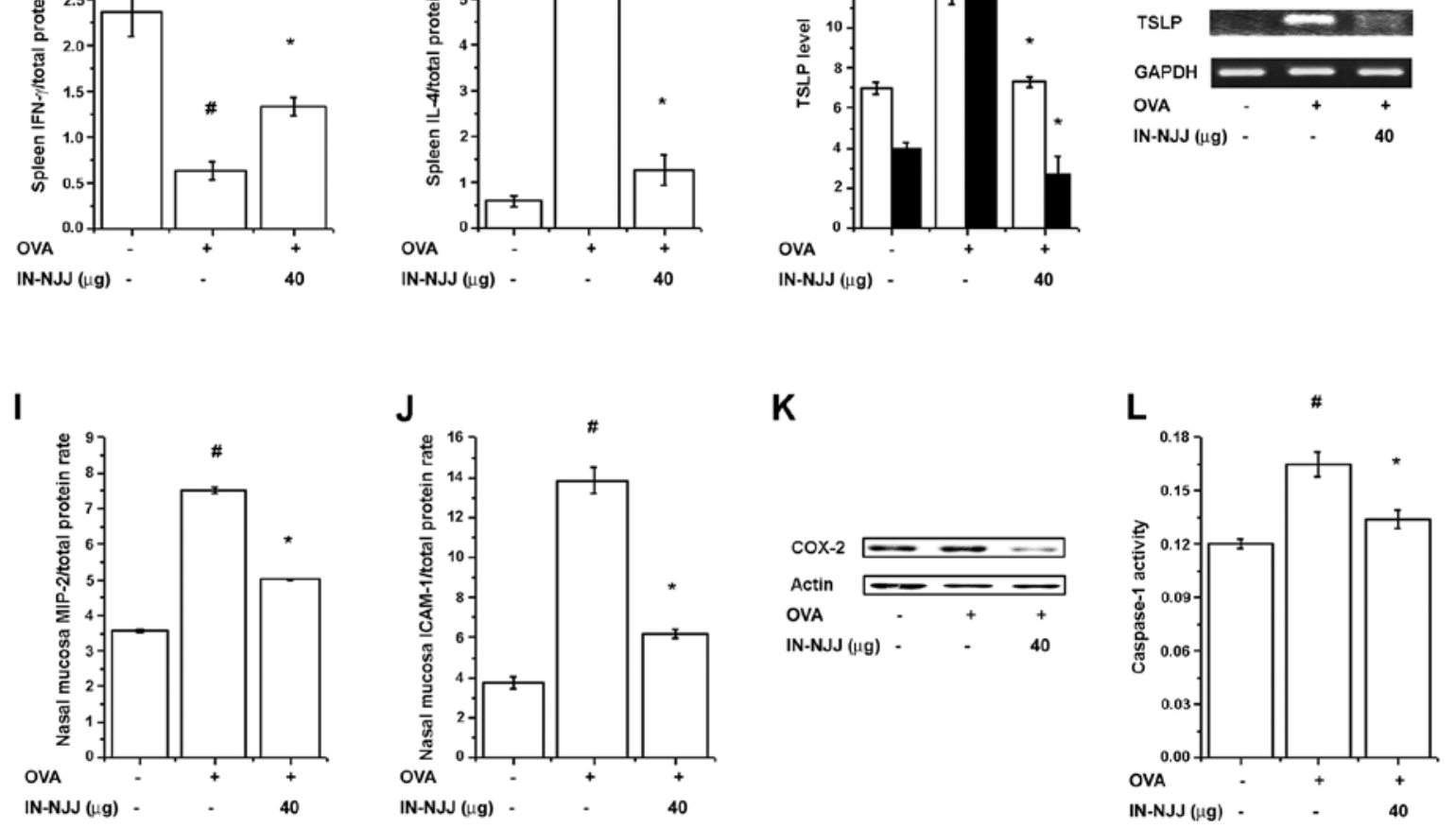

Figure 6. Effects of IN-NJJ administration in the clinical symptoms and inflammatory markers of mice with allergic rhinitis (AR). Mice were administered intranasal NJJ (IN-NJJ) for 10 days prior to the i.n. ovalbumin (OVA) challenge. (A) Number of nose, ear and eyes rubs that occurred in the 10 min after the OVA intranasal provocation. (B) Spleen weight. (C) Serum was isolated from blood and then assayed for IgE. (D) Serum histamine levels were measured by histamine assay. (E) IFN- $\gamma$ and (F) interleukin (IL)-4 levels in spleen were measured by ELISA. (G) Thymic stromal lymphopoietin (TSLP) production was measured by ELISA in the nasal mucosal tissue. (H) mRNA levels were measured by RT-PCR. (I) Macrophage-inflammatory protein-2 (MIP-2) and (J) intercellular adhesion molecule-1 (ICAM-1) levels were measured by ELISA in the nasal mucosal tissue. (K) Cyclooxygenase-2 (COX-2) protein expression was evaluated by western blot analysis. (L) The enzymatic activity of caspase-1 was examined by a caspase-1 colorimetric assay in nasal mucosal tissue of mice with AR. All parameters measured in the tissue homogenates were presented as a ratio to the total protein levels in the tissue. ${ }^{\sharp} \mathrm{P}<0.05$, significantly different from the OVA-unsensitized mice. ${ }^{~} \mathrm{P}<0.05$, significantly different from the OVA-sensitized mice. $\mathrm{N}=5$.

then contribute to the influx of mast cells, eosinophils, basophils, neutrophils and monocytes in the late response $(27,28)$. Eosinophils are innate effector cells and important in immune responses against helminth parasitic infections. They contribute to the pathology associated with allergic inflammatory conditions $(29,30)$. Eosinophil accumulation in health and disease is a hallmark characteristic of mucosal immunity and type 2 helper T cell inflammation (31). The eosinophil granule proteins play a central role in the development of rhinitis (32). IL-32 released from eosinophils has been shown to significantly increase the levels of $\operatorname{IgE}$ and inflammatory cytokines, including IL-1 $\beta$, IL-18 and GM-CSF in AR (7). Despite the absence of a Th1-cell predominant inflammation, other Th2-type inflammatory disorders, such as AR, asthma and AD have been linked with increased levels of IL-32 (33). An increase in the levels of IL-4, IgE, eosinophil cationic protein (ECP) and eosinophil infiltration by IL-32 stimulation has been demonstrated in an animal model of AR (7). These data suggest that IL-32 is an attractive target in the treatment of AR. Accordingly, we hypothesized that NJJ mediates antiallergic effects, at least partly by suppressing the production of IL-32. In this study, our results demonstrated that NJJ relieved 
the clinical symptoms of AR and decreased the levels of $\mathrm{IgE}$. NJJ reduced the levels of IL-32 and the number of infiltrating eosinophils in the mice with AR. NJJ induced a dose-related inhibition of the production and mRNA expression of IL-32 in the GM-CSF-stimulated Eol-1 cells. Therefore, we suggest that NJJ exerts anti-allergic effects. Its mechanisms of action may be associated with the decrease in IL-32 expression.

Recently, we reported that IL-32 significantly increased TSLP production in monocytes (20). TSLP is a critical regulator of innate and adaptive immune responses associated with Th2 cytokine-mediated inflammation, including AR. TSLP is expressed not only in epithelial cells but also in fibroblasts, endothelial cells and smooth muscle cells at nasal mucosal sites (34). Moon and Kim reported that TSLP is expressed in activated mast cells (18). Pyeongwee-San extract and hesperidin have been shownt o alleviate the symptoms of AR and to inhibit TSLP production and mRNA expression in mast cells $(19,35)$. AR is an IgE-mediated disease. Antigen-specific IgE binds to high affinity receptors (FceRI) on tissue mast cells, basophils and dendritic cells. The early phase response mediators induce the characteristic symptoms of AR, such as watery rhinorrhea, sneezing and itching, within minutes of allergen exposure. This is followed by the late phase response, involving the infiltration of inflammatory cells due to the release of cytokines and chemokines, resulting in congestion and inflammation (36). In this study, we demonstrated that the number of infiltrating mast cells in nasal mucosal tissue was reduced following the administration of NJJ. Therefore, it can be deduced that NJJ inhibits mast cell functions.

Caspase-1 plays a central role in innate immunity and in several important inflammatory diseases (37). Caspase-1 activation is involved in inflammation and the regulation of immune responses and differentiation (20,35). In an animal model of AR, caspase-1 activity was shown to increase compared with normal mice (35). Caspase-1 levels in allergic asthmatic patients are also higher than those in normal individuals (38). IL-1 $\beta$, IL-18, histamine and IgE have been shown to be upregulated through the activation of caspase-1 in kanamycin-administered NC/Nga mice (39). IL-32 and TSLP production are also increased by the activation of caspase-1 $(7,20)$. The activation of caspase-1 promotes COX-2-dependent inflammatory reactions (40). As previously demonstrated, COX-2 expression is reduced in caspase-1 knockout mice (40). Thus, the suppression of caspase-1 activity is associated with a reduction in inflammation. In this study, we observed that NJJ inhibited the activation of caspase-1 in the mice with AR and in the Eol-1 cells. Therefore, we hypothesized that the anti-allergic effects of NJJ may be derived from the downregulation of caspase-1 activity.

TKM has been used to treat symptoms of AR for decades and is still generally used for treatment of AR in Korea. TKM therapy is usually considered to be safe and effective in a large population. NJJ has been known for thousands of years to have antibacterial and anti-inflammatory effects. Tryptanthrin, kaempferol and indirubin are the main components of NJJ. Tryptanthrin and kaempferol have antibacterial properties (23). Kaempferol has been shown to inhibit the production of IL- 8 and tumor necrosis factor (TNF)- $\alpha$ and the infiltration of eosinophils in allergic reactions $(41,42)$. Indirubin has been shown to inhibit inflammatory reactions and allergic contact dermatitis $(43,44)$. In this study, we found that the oral and nasal administration of NJJ significantly reduced the levels of IgE, IL-4, ICAM-1, MIP-2 and COX-2 in mice with AR. Therefore, the above data suggest that the combination of various active compounds in NJJ have a synergistic effect in AR. However, the active components of NJJ should be isolated in further studies to clarify whether the components themselves may also be effective in the treatment of AR.

In conclusion, to our knowledge, we report for the first time that NJJ regulates the production of IL-32 and TSLP, as well as that of histamine, IgE, IL-4, MIP-2, ICAM-1 and COX-2 in an animal model of AR. The anti-allergic effects of NJJ are also associated with the inhibition of infiltrating inflammatory cells and the activation of caspase-1. Therefore, our data suggest the possible therapeutic application of NJJ in the treatment of allergic inflammatory diseases.

\section{Acknowledgements}

This study was supported by Naju City (2012) and the Basic Science Research Program through the National Research Foundation of Korea (NRF) funded by the Ministry of Education, Science and Technology (2012R1A1A3005103).

\section{References}

1. Varney VA,Jacobson MR, Suddarick RM, et al: Immunohistology of nasal mucosa following allergen induced rhinitis. Am Rev Respir Dis 146: 170-175, 1992.

2. Sim TC, Grant JA, Hilsmeier KA, Fukuda Y and Alam R: Pro-inflammatory cytokines in nasal secretions of allergic subjects after antigen challenge. Am J Respir Crit Care Med 149: 339-344, 1994.

3. Gupta S, Feng L, Yoshimura T, Redick J, Fu SM and Rose CE Jr: Intra-alveolar macrophage-inflammation peptide 2 induces rapid neutrophil localization in the lung. Am J Respir Cell Mol Biol 15: 656-663, 1996.

4. Durham SR, Ying S, Varney VA, et al: Cytokine messenger RNA expression for IL-3, IL-4, IL-5 and granulocyte/macrophase colony stimulating factors in the nasal mucosa after local allergen provocation. J ImmunoI 148: 2390-2394, 1992.

5. Bacon AS, McGill JI, Anderson DF, Baddeley S, Lightman SL and Holgate ST: Adhesion molecules and relationship to leukocyte levels in allergic eye disease. Invest Ophthalmol Vis Sci 39: 322-330, 1998 .

6. Tai PC and Spry CJ: The effects of recombinant granulocytemacrophage colony-stimulating factor (GM-CSF) and interleukin-3 on the secretory capacity of human blood eosinophils. Clin Exp Immunol 80: 426-434, 1990.

7. Jeong HJ, Shin SY, Oh HA, Kim MH, Cho JS and Kim HM: IL-32 up-regulation is associated with inflammatory cytokine production in allergic rhinitis. J Path 224: 553-563, 2011.

8. Marcondes AM, Mhyre AJ, Stirewalt DL, Kim SH, Dinarello CA and Deeg HJ: Dysregulation of IL-32 in myelodysplastic syndrome and chronic myelomonocytic leukemia modulates apoptosis and impairs NK function. Proc Natl Acad Sci USA 105: 2865-2870, 2008.

9. Bae S, Kang D, Hong J, et al: Characterizing antiviral mechanism of interleukin-32 and a circulating soluble isoform in viral infection. Cytokine 58: 79-86, 2012.

10. Heinhuis B, Koenders MI, van Riel PL, et al: Tumour necrosis factor alpha-driven IL-32 expression in rheumatoid arthritis synovial tissue amplifies an inflammatory cascade. Ann Rheum Dis 70: 660-667, 2011.

11. Kim SH, Han SY, Azam T, Yoon DY and Dinarello CA: Interleukin-32: a cytokine and inducer of TNF alpha. Immunity 22: 131-142, 2005.

12. Netea MG, Azam T, Ferwerda G, et al: IL-32 synergizes with nucleotide oligomerization domain (NOD) 1 and NOD2 ligands for IL-1beta and IL-6production through a caspase 1-dependent mechanism. Proc Natl Acad Sci USA 102: 16309-16314, 2005. 
13. Nishida A, Andoh A, Shioya M, Kim-Mitsuyama S, Takayanagi A and Fujiyama Y: Phosphatidylinositol 3-kinase/Akt signaling mediates interleukin-32alpha induction in human pancreatic periacinar myofibroblasts. Am J Physiol Gastrointest Liver Physiol 294: G831-G838, 2008.

14. Heinhuis B, Koenders MI, van den Berg WB, Netea MG, Dinarello CA and Joosten LA: IL-32 contains a typical alphahelix bundle structure that resembles the focal adhesion targeting region of focal adhesion kinase-1. J Biol Chem 287: 5733-5743, 2012.

15. Ying S, O'Connor B, Ratoff J, et al: Expression and cellular provenance of thymic stromal lymphopoietin and chemokines in patients with severe asthma and chronic obstructive pulmonary disease. J Immunol 181: 2790-2798, 2008.

16. Soumelis V, Reche PA, Kanzler H, et al: Human epithelial cells trigger dendritic cell mediated allergic inflammation by producing TSLP. Nat Immunol 3: 673-680, 2002.

17. Bunyavanich S, Melen E, Wilk JB, et al: Thymic stromal lymphopoietin (TSLP) is associated with allergic rhinitis in children with asthma. Clin Mol Allergy 9: 1, 2011.

18. Moon PD and Kim HM: Thymic stromal lymphopoietin is expressed and produced by caspase-1/NF- $\kappa \mathrm{B}$ pathway in mast cells. Cytokine 54: 239-243, 2011.

19. Song YH, Nam SY, Choi Y, Kim JH, Kim YS and Jeong HJ: Socioeconomic impact of traditional Korean medicine, Pyeongwee-San (KMP6) as an anti-allergic inflammatory drug. TANG 2: 52-60, 2012

20. Jeong HJ, Nam SY, Oh HA, et al: Interleukin-32-induced thymic stromal lymphopoietin plays a critical role in macrophage differentiation through the activation of caspase-1 in vitro. Arthritis Res Ther 14: R259, 2012.

21. Iwaki K, Satomi KM, Kohno K, Ushio S and Fukuda S Antimicrobial activity of Polygonum tinctorium Lour: extract against oral pathogenic bacteria. J Nat Med 60: 121-125, 2006.

22. Satomi KM, Kimoto T, Micallef MJ, et al: Prevention of azoxymethane-induced intestinal tumors by a crude ethyl acetate-extract and tryptanthrin extracted from Polygonum tinctorium Lour. Anticancer Res 21: 3295-3300, 2001.

23. Kataoka M, Hirata K, Kunikata T, et al: Antibacterial action of tryptanthrin and kaempferol, isolated from the indigo plant (Polygonum tinctorium Lour.), against Helicobacter pyloriinfected Mongolian gerbils. J Gastroenterol 36: 5-9, 2001.

24. Kim HM, Hong DR, and Lee EH: Inhibition of mast celldependent anaphylactic reactions by the pigment of Polygonum tinctorium (Chung-Dae) in rats. Gen Pharmacol 31: 361-365, 1998.

25. Miyata M, Hatsushika K, Ando T, et al: Mast cell regulation of epithelial TSLP expression plays an important role in the development of allergic rhinitis. Eur J Immunol 38: 1487-1492, 2008.

26. Han NR, Kim HM and Jeong HJ: Thymic stromal lymphopoietin is regulated by the intracellular calcium. Cytokine 59: 215-217, 2012.

27. Spada CS, Nieves AL,Krauss AH and Woodward DF: Comparison of leukotriene B4 and D4 effects on human eosinophil and neutrophil motility in vitro. J Leukoc Biol 55: 183-191, 1994.
28. Sugimoto H, Shichijo M, Iino T et al: An orally bioavailable small molecule antagonist of CRTH2, ramatroban (BAY u3405), inhibits prostaglandin D2-induced eosinophil migration in vitro. J Pharmacol Exp Ther 305: 347-352, 2003.

29. Melvin TA and Ramanathan M Jr: Role of innate immunity in the pathogenesis of allergic rhinitis. Curr Opin Otolaryngol Head Neck Surg 20: 194-198, 2012.

30. Isobe $\mathrm{Y}$, Kato T and Arita M: Emerging roles of eosinophils and eosinophil-derived lipid mediators in the resolution of inflammation. Front Immunol 3: 270, 2012.

31. Moshkovits I, Shik D, Itan M, et al: CMRF35-like molecule 1 (CLM-1) regulates eosinophil homeostasis by suppressing cellular chemotaxis. Mucosal Immunol: Jul 3, 2013 (Epub ahead of print). doi: 10.1038/mi.2013.47.

32. Bystrom J, Patel SY, Amin K and Bishop-Bailey D: Dissecting the role of eodinophil cationic protein in upper airway disease. Curr Opin Allergy Clin Immunol 12: 18-23, 2012.

33. Soyka MB, Treis A, Eiwegger T, et al: Regulation and expression of IL-32 in chronic rhinosinusitis. Allergy 67: 790-798, 2012.

34. Nomura K, Kojima T, Fuchimoto J, Obata K, Keira T, Himi T and Sawada N: Regulation of interleukin-33 and thymic stromal lymphopoietin in human nasal fibroblasts by proinflammatory cytokines. Laryngoscope 122: 1185-1192, 2012.

35. Oh HA, Kim HM and Jeong HJ: Alleviation of allergic rhinitis symptoms with Pyeongwee-San extract (KMP6). Immunopharmacol Immunotoxicol 34: 135-142, 2012.

36. Vashisht $P$ and Casale T: Omalizumab for treatment of allergic rhinitis. Expert Opin Biol Ther 13: 933-945, 2013.

37. Sollberger G, Strittmatter GE, Garstkiewicz M, Sand J and Beer HD: Caspase-1: the inflammasome and beyond. Innate Immun: May 15, 2013 (Epub ahead of print).

38. Grzegorczyk J, Kowalski ML, Pilat A and Iwaszkiewicz J: Increased apoptosis of peripheral blood mononuclear cells in patients with perennial allergic asthma/rhinitis: relation to serum markers of apoptosis. Mediators Inflamm 11: 225-233, 2002.

39. Han NR, Kim HM and Jeong HJ: Kanamycin activates caspase-1 in NC/Nga mice. Exp Dermatol 20: 659-663, 2011.

40. Cunha TM, Talbot J, Pinto LG, et al: Caspase-1 is involved in the genesis of inflammatory hypernociception by contributing to peripheral IL-1 $\beta$ maturation. Mol Pain 6: 63, 2010.

41. Lee EJ, Ji GE and Sung MK: Quercetin and kaempferol suppress immunoglobulin E-mediated allergic inflammation in RBL-2H3 and Caco-2 cells. Inflamm Res 59: 847-854, 2010.

42. Gong JH, Shin D, Han SY, Kim JL and Kang YH: Kaempferol suppresses eosionphil infiltration and airway inflammation in airway epithelial cells and in mice with allergic asthma. J Nutr 142: 47-56, 2012

43. Kunikata T, Tatefuji T, Aga H, Iwaki K, Ikeda M and Kurimoto M Indirubin inhibits inflammatory reactions in delayed-type hypersensitivity. Eur J Pharmacol 410: 93-100, 2000

44. Kim MH, Choi YY, Yang G, Cho IH, Nam D and Yang WM: Indirubin, a purple 3,2-bisindole, inhibited allergic contact dermatitis via regulating Thelper(Th)-mediated immune system in DNCB-induced model. J Ethnopharmacol 145: 214-219, 2013. 